$\xi=2$ 줄

\title{
Comparative evaluation of antimicrobial activity of biodentine and MTA against e. faecalis an in vitro study
}

\author{
Jerin Jose ${ }^{1 *}$, Shoba $K^{2}$, Nithya Tomy ${ }^{3}$, Shibu Aman ${ }^{3}$ \\ ${ }^{1}$ Junior Resident, Department of Conservative Dentistry and Endodontics, Government Dental College, Kottayam \\ ${ }^{2}$ Professor and Head of the Department, Department of Conservative Dentistry and Endodontics, Government Dental College, Kottayam \\ ${ }^{3}$ Junior Resident, Department of Pedodontics and Preventive Dentistry, Kannur Dental College, Kannur \\ ${ }^{3}$ Assistant Professor, Department of Conservative Dentistry and Endodontics, Government Dental College, Kottayam \\ *Corresponding author E-mail:jerjo888@gmail.com
}

\begin{abstract}
Background: Surgical endodontic therapy is the prescribed treatment of choice whenever the non-surgical options fail which includes the use of a root end filling material. E. faecalis is the most prevalent organism associated with the treatment failed cases

Aim: The purpose of the present study was to evaluate and compare the antimicrobial efficacy of Biodentine and MTA as root end filling material and root repair material against E. faecalis.

Settings and Design: Comparative invitro study was conducted at Tropical Institute of Ecological Sciences, Kottayam under Mahatma Gandhi University, Kottayam, Kerala.

Materials and Methods: The antimicrobial activity was determined using agar diffusion test. Freshly mixed MTA and Biodentine was added to respective wells and incubated. The zone of inhibition of growth was recorded.

Statistical analysis: The data analysis was performed using Students ' $t$ ' test

Results: Statistically significant difference was found between Biodentine and MTA in against E. faecalis growth.

Conclusions: Biodentine was found to have superior antibacterial activity against E. faecalis than MTA. Hence it can be a better alternative to MTA as root end filling and root repair material.

Keywords: Biodentine; MTA; E. Faecalis; Root End Filling Material; Root Repair Material
\end{abstract}

\section{Introduction}

Bacteria are the prime etiological agents in the development and progression of pulpal and periapical diseases (Kakehashi et al. 1965). When orthograde or non-surgical root canal treatment gets failed, surgical endodontic therapy involving resecting a portion of the root apex with retrograde obturation is indicated to retain the tooth (Gutmann and Harrison 1999). The root-end filling material functions as either enhancing the existing seal or contributing to a new apical seal to an otherwise unobturated root canal.

Certain microorganisms like E. faecalis is repeatedly recovered from failed root canal treated cases or retreatment cases. Its prevalence in such infections is high because of the virulence factors possessed by the organism (Stuart et al. 2006). Hence, the antimicrobial effects of root end filling material may play an important role in the healing process and treatment outcome.

Numerous biomaterials are used to obtain the three-dimensional seal at the apical region during the surgical treatment. MTA is a biomaterial with an excellent biocompatibility and adequate sealing property (Kim and Kratchman 2006). Biodentine is a novel calcium silicate bioactive material with some superior properties when comparing to other materials. As Biodentine is recently introduced, studies regarding comparison of its antimicrobial activity with MTA are minimal.
The aim of the study is to compare the antibacterial activity of Biodentine and MTA as root end filling material and root repair material against E. faecalis.

\section{Subjects and methods}

This invitro study was conducted at the Tropical Institute of Ecological Sciences, Velloor, Kottayam and Institutional scientific committee, Government Dental College; Kottayam gave the approval for this in-vitro study. MTA Angelus was used for testing and it is manufactured by Angelus, Londrina-PR, Brazil. Manufacturer of Biodentine is Septodont, St. Maur-des-Fossés, France. The antibacterial activities of the materials were evaluated using agar diffusion test. The Enterococcus faecalis strain used for agar diffusion test was ATCC 29212.

The method of choice was agar well diffusion method. From prepared culture, $100 \mu 1$ of E. faecalis suspension was taken and inoculated in $130 \mathrm{~mm}$ culture plates with previously set layers of Mueller Hinton Agar. Sterile spreader was used for performing the inoculations across media. After that two uniform wells of $6 \mathrm{~mm}$ diameter and depth were made in the agar plate containing cultures of E. faecalis.

$50 \mathrm{mg}$ of MTA and Biodentine were taken and mixed according to the manufacturer's instructions. $200 \mu$ l of MTA and Biodentine were added to the respective wells on the plate and were incubated for 24 hours at $37^{\circ} \mathrm{C}$ in an incubator. After incubation period, plate was checked for the zones of inhibition of bacterial growth. The 
diameter of the zones of inhibition of bacterial growth obtained for two groups against E. faecalis were recorded in centimeter (Fig. 1). Agar diffusion test had performed 6 times for obtaining significant results.

\section{Results}

The results were statistically analyzed using computer software Statistical Package for Social Sciences (SPSS) version 16. Graph
1 shows diameters of the zone of inhibition against $\mathrm{E}$. faecalis growth attained by MTA and Biodentine. Table 1 shows mean diameter of the zone of inhibition against E. faecalis growth obtained for MTA was $3.250 \mathrm{~cm}$ and that obtained for Biodentine was $3.983 \mathrm{~cm}$. Also table 1 shows that there was significant difference exist between the diameters of zone of inhibition of bacterial growth obtained for MTA and Biodentine against E. faecalis $(p<0.05)$. This finding was found out using student's ' $t$ ' test.

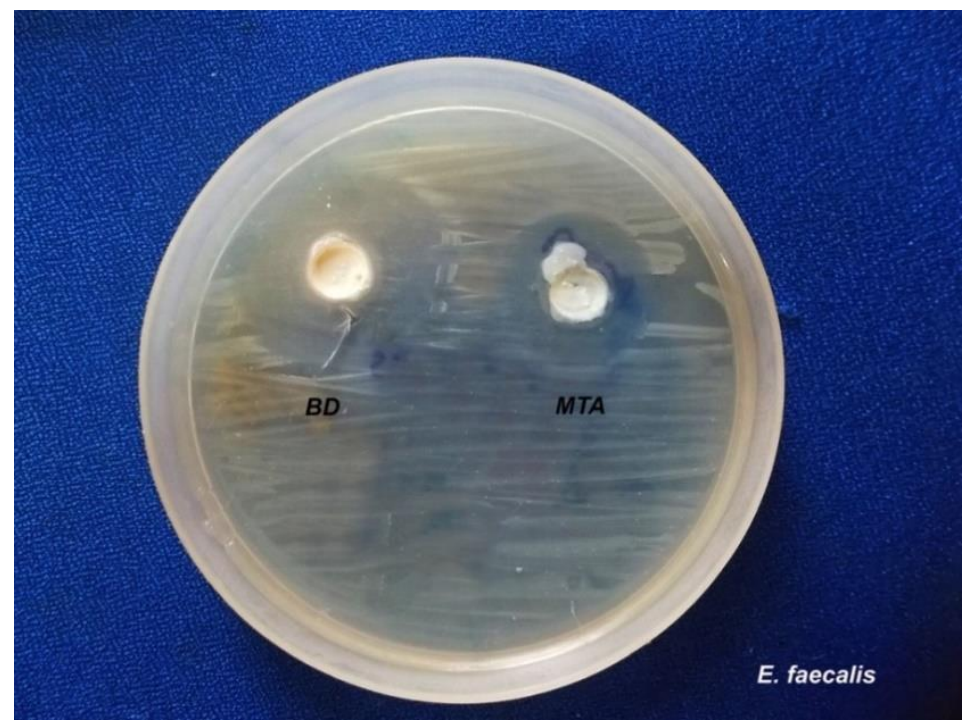

Fig. 1: Agar Diffusion Test

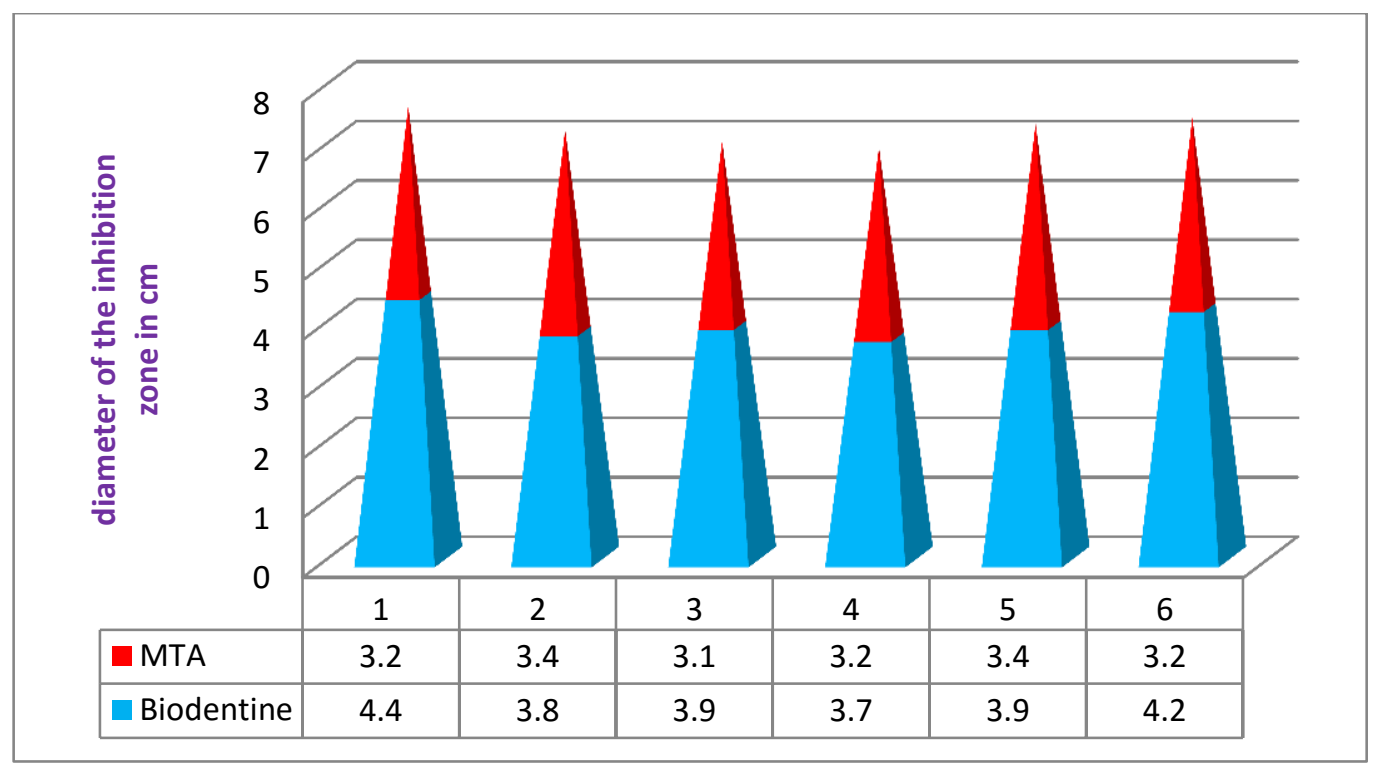

Graph 1: Comparison of Diameter of Zone of Inhibition Obtained for MTA and Biodentine.

Table 1: Student's T Test for Mean Diameter of Zone of Inhibition of Bacterial Growth Comparing Antimicrobial Activity of Two Bioactive Materials

\begin{tabular}{|c|c|c|c|c|c|c|c|c|c|}
\hline \multirow{2}{*}{ Organism } & \multirow{2}{*}{ Material } & \multirow{2}{*}{ Mean $(\mathrm{cm})$} & \multirow{2}{*}{ Std. deviation } & \multirow{2}{*}{ Std. error of mean } & \multicolumn{2}{|c|}{ Levene's Test for Equality of Variances } & \multicolumn{3}{|c|}{ t-test for Equality of Means } \\
\hline & & & & & $\mathrm{F}$ value & Sig & $\mathrm{t}$ & df & Sig. \\
\hline \multirow{2}{*}{ E. faecalis } & MTA & 3.250 & 0.122 & 0.050 & \multirow{2}{*}{3.861} & \multirow{2}{*}{0.078} & -6.173 & 10 & 0.000 \\
\hline & Biodentine & 3.983 & 0.263 & 0.107 & & & -6.173 & 7.058 & 0.000 \\
\hline
\end{tabular}

\section{Discussion}

An ideal root repair material should have antibacterial properties either they should be bactericidal or bacteriostatic. Also root end filling materials filling material should be antimicrobial in nature. This is because many bacteria which are the major constituent of primary infection can be present along with the therapy resistant organisms in the areas of persistent infection. In this in vitro study, we were trying to evaluate and compare antibacterial efficacy of MTA with a newer root end filling and root repair material, Bio- dentine. One of the most predominant and prevalent organisms associated with therapy resistant cases is E. faecalis (Stuart et al. 2006). Majority of the cases selecting for surgical endodontic therapy include treatment failure cases. Therefore organism selected for testing the antibacterial activity of MTA and Biodentine was E. faecalis.

Agar diffusion test was opted because it is one of the efficient and commonly used methods used for evaluating the antimicrobial activity of materials and the efficacy of the method had already proven by various antimicrobial studies (Lui et al. 2004). The 
findings obtained from agar diffusion test are usually influenced by the ability of the material to diffuse across the medium (Fraga et al. 1996). The factors that affect the results of agar diffusion tests are selection of the agar medium and microorganisms, standardization of inoculation density and incubation and reading point of the zones of inhibition (Sipert et al. 2005). In the present study, we used freshly mixed cements of MTA and biodentine for agar diffusion test because antimicrobial activity of different materials may be related to the degree of setting (Cobankara et al. 2004).

There are many materials which are used as root end filling material and among them MTA fulfills major criteria's and considered as an ideal material for root repair and root end filling. MTA has its advantages like regenerative and osteogenic potential, good marginal sealing ability, biocompatibility and bioactivity (Saunders et al. 2008). The push-out bond strength had shown to be increase gradually over time. The antimicrobial activity of MTA against $\mathrm{E}$. faecalis and $\mathrm{P}$. aeruginosa had demonstrated in previous studies (Stowe et al. 2004) Antibacterial properties of this calcium silicate material can be attributed to its alkalinity (Fridland and Rosado 2003). The $\mathrm{pH}$ value of the freshly mixed MTA is 10.2, which increases upto 12.5 after 3 hours. This increase in the $\mathrm{pH}$ value might be due to calcium hydroxide release during the hydration process (Fridland and Rosado 2005).

Biodentine is a recently introduced bioactive dentine substitute based on "Active Biosilicate Technology". The properties like biocompatibility and bioactivity, sealing ability and regenerative potential similar to that of MTA but setting time and handling properties are better when comparing to MTA (Kokate and Pawar 2012). Antibacterial properties of biodentine are closely associated with $\mathrm{pH}$ and calcium release. The $\mathrm{pH}$ of cement rises to 12.5 during setting and a basification of near-by areas occur. The microbial growth is inhibited at this $\mathrm{pH}$ which may lead to the disinfection of the surrounding areas. Also the properties such as compressive strength, elasticity modulus and microhardness of Biodentine are more similar to that of natural dentine, hence proposed to be ideal root end filling and root repair material (Han and Okiji 2011)

Our results demonstrated that both materials possess antimicrobial activity which had been substantiated by the formation of zones of inhibition. The mean diameter of the zone of inhibition obtained for MTA was $3.25 \mathrm{~cm}$ and that obtained for Biodentine was $3.98 \mathrm{~cm}$. It was evident that Biodentine achieved a larger zone of inhibition when compared to MTA which was an indicative of its greater antibacterial activity. This was statistically significant also. The results of our studies are in parallel with the studies conducted by Tanomaru- Filho et al. where they had obtained a zone of inhibition of $16 \mathrm{~mm}$ for MTA against E. faecalis. Also the present study shows a positive correlation with a recent study conducted by Bhavana et al. in 2015. They demonstrated that the mean diameter of zone of inhibition attained by MTA against $\mathrm{E}$. faecalis was $2.6 \mathrm{~cm}$ and that by Biodentine was $3.1 \mathrm{~cm}$.

\section{Conclusion}

From the present study it can be concluded that Biodentine possesses superior antibacterial activity against E. faecalis than MTA which is significant. Excellent antibacterial property against highly pathogenic organism like E. faecalis along with other properties such as stability, less solubility, non-resorbable nature, better handling properties, fast setting time and good sealing ability make Biodentine, a better alternative for root repair and root end filling when comparing to MTA. As previous studies comparing the antibacterial activity of Biodentine and MTA are comparatively fewer in number, further in-vitro and ex-vivo studies are recommended before establishing this novel calcium silicate material as an ideal root repair and root end filling material.

\section{References}

[1] Kakehashi S, Stanley HR, Fitzgerald RJ. The effects of surgical exposures of dental pulps in germ-free and conventional laboratory rats. Oral Surg Oral Med Oral Pathol. 1965; 20:340-9. http://dx.doi.org/10.1016/0030-4220(65)90166-0.

[2] Fraga RC, Siqueira JF Jr, de Uzeda M. In vitro evaluation of antibacterial effects of photo cured glass ionomer liners and dentin bonding agents during setting. J Prosthet Dent. 1996; 76:483-486. http://dx.doi.org/10.1016/S0022-3913(96)90005-0.

[3] Gutmann and Harrison, Gutmann's surgical endodontics, 1999

[4] Fridland M, Rosado R. Mineral trioxide aggregate (MTA) solubility and porosity with different water-to-powder ratios. J Endod. 2003; 29(12):814- http://dx.doi.org/10.1097/00004770-200312000-00007.

[5] Cobankara FK, Altinoz HC, Ergani O, Kav K, Belli S. In vitro antibacterial activities of root canal sealers by using two different methods. J Endod. 2004; 30:57-60 http://dx.doi.org/10.1097/00004770-200401000-00013.

[6] Lui JN, Sae-Lim V, Song KP, Chen NN. In vitro antimicrobial effect of chlorhexidine impregnated gutta percha points on Enterococcus feacalis. Int Endod J2004; 37:105-113 http://dx.doi.org/10.1111/j.0143-2885.2004.00734.x.

[7] Stowe TJ, Sedgley CM, Stowe B, Fenno JC. The effects of chlorhexidine gluconate $(0.12 \%)$ on antimicrobial properties of toothcolored ProRoot mineral trioxide aggregate. J Endod 2004; 30:429 431. http://dx.doi.org/10.1097/00004770-200406000-00013.

[8] Fridland M, Rosado R. MTA solubility: a long term study. J Endod. 2005 ; $31(5): 376-9$ http://dx.doi.org/10.1097/01.DON.0000140566.97319.3e.

[9] Sipert CR, Hussne RP, Nishiyama CK, Torres SA. In vitro antimicrobial activity of Fill Canal, Sealapex, Mineral Trioxide Aggregate, Portland cement and EndoRez. Int Endod J. 2005;38:539-543 http://dx.doi.org/10.1111/j.1365-2591.2005.00984.x.

[10] Kim S, Kratchman S. Modern endodontic surgery concepts and practice: a review. J Endod 2006; 32:601-623. http://dx.doi.org/10.1016/j.joen.2005.12.010.

[11] Stuart CH, Schwartz SA, Beeson TJ, Owatz CB, Enterococcus faecalis: its role in root canal treatment failure and current concepts in $\begin{array}{llll}\text { retreatment. } & \mathrm{J} & \text { Endod.2006; } & \end{array}$ http://dx.doi.org/10.1016/j.joen.2005.10.049.

[12] Saunders WP. A prospective clinical study of periradicular surgery using Mineral trioxide aggregate as a root-end filling. J Endod. 2008; 34(6):660-664. http://dx.doi.org/10.1016/j.joen.2008.03.002.

[13] Bronnec F. Biodentine: a dentin substitute for the repair of root perforations, apexification and retrograde root filling. J Endod 2010; 36(3):400-413.

[14] Han L, Okiji T. Uptake of calcium and silicon released from calcium silicate based endodontic materials into root canal dentine. Int Endod J 2011; 44:1081-7. http://dx.doi.org/10.1111/j.13652591.2011.01924.x.

[15] Kokate SR, Pawar AM. An in vitro comparative stereomicroscopic evaluation of marginal seal between MTA, Glass Inomer Cement $\&$ BiodentineTM as root end filling materials using $1 \%$ methylene blue as tracer. Endod 2012; 2:36-42. 